\begin{tabular}{|c|l|}
\hline Title & $\begin{array}{l}\text { Carrier-transfer dynamics between neutral and charged excitonic states in a single quantum dot probed with second- } \\
\text { order photon correlation measurements }\end{array}$ \\
\hline Author(s) & Nakajima, H.; Kumano, H.; lijima, H.; Odashima, S.; Suemune, I. \\
\hline Citation & $\begin{array}{l}\text { Physical Review B, 88(4), 045324_1-045324.6 } \\
\text { https://doi.org/10.1103/PhysRevB.88.045324 }\end{array}$ \\
\hline Issue Date & 2013-07-31 \\
\hline Doc URL & http://hdl.handle.net/2115/59634 \\
\hline Rights & O2013A merican Physical Society \\
\hline Type & article \\
\hline File Information & Nakajima_PRB13.pdf \\
\hline
\end{tabular}

Instructions for use 


\title{
Carrier-transfer dynamics between neutral and charged excitonic states in a single quantum dot probed with second-order photon correlation measurements
}

\author{
H. Nakajima, ${ }^{1,2, *}$ H. Kumano, ${ }^{1}$ H. Iijima, ${ }^{1}$ S. Odashima, ${ }^{1}$ and I. Suemune ${ }^{1}$ \\ ${ }^{1}$ Research Institute for Electronic Science, Hokkaido University, N21W10, Kita-ku, Sapporo 001-0021, Japan \\ ${ }^{2}$ Research Fellow of the Japan Society for the Promotion of Science, 1-8, Chiyoda-ku, Tokyo 102-8472, Japan
}

(Received 2 May 2013; published 31 July 2013)

\begin{abstract}
We report a comprehensive investigation of carrier-transfer dynamics in a single InAs quantum dot (QD) based on second-order photon correlation measurements. The experimentally obtained auto and crosscorrelation functions as well as photoluminescence intensities are successfully explained on the basis of a series of rate equations with common excitation and also single-carrier-capture/escape rates to/from a QD. This approach enables us to understand the carrier-transfer dynamics responsible for the stability of a quantum two-level system formed in a single QD under various excitation conditions. We clarify that the transition between neutral and charged excitonic states is suppressed by one order of magnitude under quasiresonant excitation.
\end{abstract}

DOI: 10.1103/PhysRevB.88.045324

PACS number(s): 68.65.Hb, 78.55.Cr, 42.50.Ar

\section{INTRODUCTION}

A single semiconductor quantum dot (QD) forms discrete energy levels owing to its three-dimensional confinement similar to isolated atoms and exhibits a distinct quantum nature that can be tailored for versatile quantum information applications. Excitonic species bound inside a QD constitute discrete emission lines ${ }^{1}$ in the photoluminescence (PL) spectrum and inherently provide population and spin correlations between the lines. Thus QDs are good candidates for solid-state single-photon or entangled photon-pair emitters in quantum cryptography ${ }^{2}$ and linear optical computing. ${ }^{3}$ To date, the generation of single photons ${ }^{4-8}$ and entangled photon pairs ${ }^{9-16}$ have been demonstrated by evaluating the second-order photon correlation function $g^{(2)}(\tau)$. In addition, correlation function analysis is a powerful tool for evaluating relatively fast carrier dynamics, which appears as blinking or spectral diffusion, owing to its high time resolution of nanosecond order. ${ }^{17-30}$ Thus $g^{(2)}(\tau)$ contains rich information on microscopic carriertransfer dynamics involving a single $\mathrm{QD}$, which is valuable for maximizing a secure key rate or gate time for coherent control.

Using the correlation functions measured by interline ${ }^{17-20}$ and intraline spectral filtering, ${ }^{25-30}$ the carrier-transfer dynamics occurring in the vicinity of and across a QD interface have been discussed. The former affects the energy eigenvalues of electronic states in the QD by inducing temporal fluctuations of the field, and the latter alters the charge state in the QD. Carrier-transfer dynamics is likely to be influenced by the population of the states involved. Experimentally, the correlation functions reflecting population dynamics in a specific energy window were indeed shown to be sensitive to the excitation power. ${ }^{18,25,28}$ Furthermore, strong modification of the dynamics by changing the excitation energy has been recently reported ${ }^{27}$ Therefore an analytical treatment valid for a wide range of excitation conditions is required for the detailed understanding of carrier-transfer dynamics affecting electronic states in a QD.

In this paper, the carrier-transfer dynamics resulting in charge state fluctuations in a single QD was comprehensively studied by employing a series of rate equations, which are applicable to various excitation powers and energies. Consequently, we have revealed that single-electron capture to a QD dominates the dynamics of charge state fluctuations, and under quasiresonant excitation, the capture rate is strongly suppressed by one-order of magnitude.

\section{EXPERIMENTAL PROCEDURE}

We studied an InAs QD grown on a GaAs (100) substrate by metal-organic molecular beam epitaxy. The density of the QDs was estimated to be $\sim 3.5 \times 10^{9} \mathrm{~cm}^{-2}$. After fabricating pillar-shaped structures (400-nm diameter) by dry etching, the $\mathrm{Ag}$ was evaporated as a photon reflector, which gives a photon extraction efficiency as high as $\sim 20 \%$. $^{31,32}$ Further details about the fabrication processes can be found in Refs. 32 and 33 .

For optical excitation, a continuous-wave (CW) Ti:Sa laser (operating at 800-930 $\mathrm{nm}$ ) or a mode-locked one (repetition rate of $76 \mathrm{MHz}$, pulse duration of $5 \mathrm{ps}$, operating at $800 \mathrm{~nm}$ ) was used. The excitation lasers were selectively focused with an objective lens $(\mathrm{NA}=0.4)$ onto a single-pillar structure containing QDs at $20 \mathrm{~K}$. For single QD spectroscopy, we used a $0.64-\mathrm{m}$ triple monochromator with a grating of 1800 grooves/mm and a liquid-nitrogen-cooled Si chargecoupled-device detector. Second-order photon correlation measurements were performed with a conventional Hanbury Brown and Twiss (HBT) setup. ${ }^{34}$ The optical path from the QD was divided by a 50/50 beam splitter into two orthogonal paths, and the emitted photons were sent to band-pass filters having a FWHM of $0.5 \mathrm{~nm}$ on each path to select the $\alpha(\beta)$ emission line, followed by single-photon counting modules (SPCMs) to generate start (stop) TTL pulses. Coincidence counts measured with [start, stop] $=[\alpha, \beta]$ configurations were built up as a function of delay time $\tau$ with 100-ps time bins, and correlation functions $g^{(2)}(\tau)$ were obtained after accumulation over $1 \sim 5 \mathrm{~h}$ using a multichannel scaler without background subtraction. All the measured $g^{(2)}(\tau)$ were normalized by raw coincidence counts at $\tau \sim 300 \mathrm{~ns}$, which is well separated from zero delay time and is much longer than the $\mathrm{QD}$ lifetime $\sim 1 \mathrm{~ns}$.

\section{RESULTS AND DISCUSSION}

\section{A. Optical properties of a single quantum dot}

Figure 1(a) shows a micro-PL ( $\mu$-PL) spectrum from an InAs QD under CW excitation. The excitation wavelength 
(a)

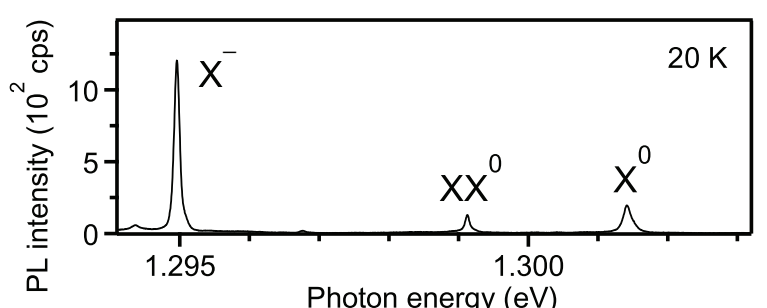

(b)

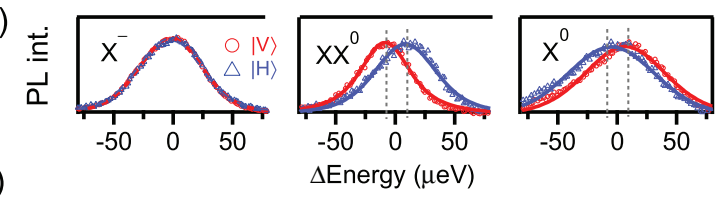

(c)

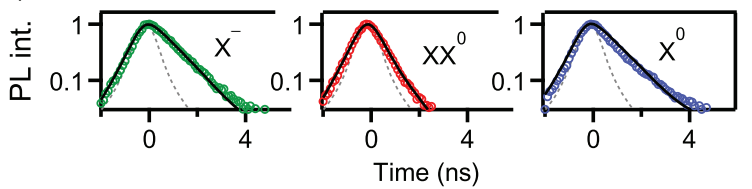

FIG. 1. (Color online) (a) $\mu$-PL spectrum from a InAs QD at $20 \mathrm{~K}$ with an excitation power of $65 \mu \mathrm{W} . X^{0}, X X^{0}$, and $X^{-}$ emissions were observed. (b) $\mu$-PL spectrum with $H$ (V) polarization detection denoted by red circles (blue triangles). Origins of the horizontal axes are set at the mean energies for each emission line. (c) Temporal profiles for the three emission lines. Dotted lines indicates the response function of our measurement system. In the present QD, single exponential population decay is observed. Thus the bright to dark state transitions (Refs. 35 and 36) are discarded in this study.

was $800 \mathrm{~nm}$, which corresponds to photoexcitation above the band edge of the GaAs barrier (nonresonant excitation). Three emission lines were prominently observed. On the basis of fine-structure splitting (FSS) and the transition energies, we assigned the observed lines to a neutral exciton $\left(X^{0}\right.$, $E_{X^{0}}=1.3014 \mathrm{eV}$, FWHM $\left.\sim 80 \mu \mathrm{eV}\right)$, neutral biexciton $\left(X X^{0}, E_{X^{0}}=1.2991 \mathrm{eV}, \mathrm{FWHM} \sim 50 \mu \mathrm{eV}\right)$, and negatively charged exciton $\left(X^{-}, E_{X^{-}}=1.2950 \mathrm{eV}, \mathrm{FWHM} \sim\right.$ $60 \mu \mathrm{eV})$. The binding energies of $X X^{0}$ and $X^{-}$, which are experimentally defined by $E_{\mathrm{X}^{0}}-E_{\mathrm{XX}^{0}}\left(E_{\mathrm{X}^{-}}\right),{ }^{37}$ correspond to +2.3 and $+6.4 \mathrm{meV}$, respectively. These are good in agreement with previously reported values with same material system. ${ }^{38}$ The excitation power was $65 \mu \mathrm{W}$, giving rise to $\sim 30 \%$ of the saturated PL intensity for the $X^{-}$ emission.

Figure 1(b) shows the polarization-resolved $\mu$-PL spectra for the three emission lines. The measured spectrum with vertical- and horizontal-polarization detection are shown by red circles and blue triangles, respectively, and were well fitted to Lorentz functions. Both $X^{0}$ and $X X^{0}$ lines have an FSS of $\sim 20 \mu \mathrm{eV}$, while the FSS of $X^{-}$is absent ${ }^{39}$ within the spectral resolution of our system $(\sim 5 \mu \mathrm{eV})$. The temporal behaviors of the PL intensities under pulsed excitation are shown in Fig. 1(c). Single-exponential decays convolved with a system response function of $\sim 1 \mathrm{~ns}$ (dotted line) reproduce the measured decay profiles for the $X^{0}, X X^{0}$, and $X^{-}$lines with lifetimes of $0.77,0.38$, and $0.75 \mathrm{~ns}$, respectively, as indicated by solid lines.

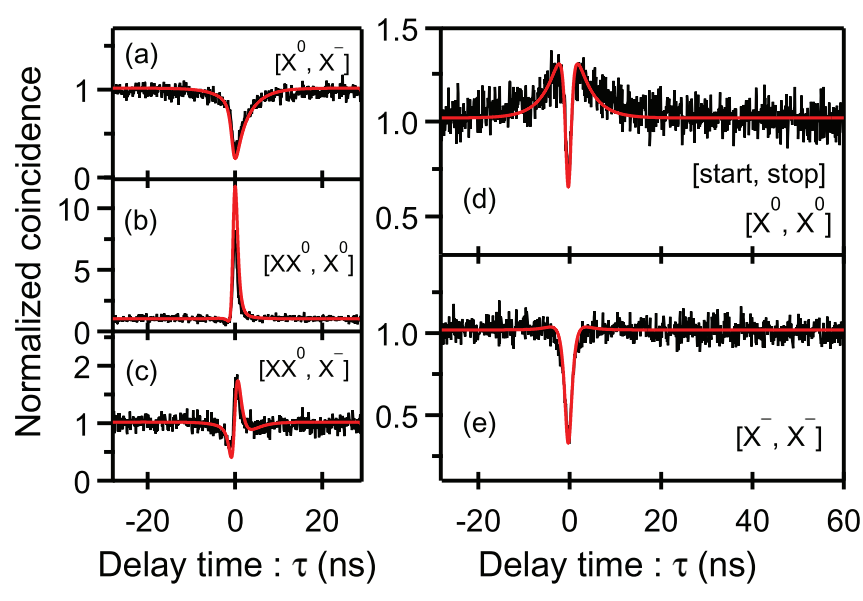

FIG. 2. (Color online) Second-order photon correlation functions $g^{(2)}(\tau)$ measured with five [start, stop] configurations. Red lines are simulation results based on a rate-equation analysis formulated from the five-level model illustrated in Fig. 3.

\section{B. Carrier-transfer dynamics}

Figure 2 shows the measured second-order correlation functions. The cross-correlation functions obtained from [start, stop $]=$ (a) $\left[X^{0}, X^{-}\right]$, (b) $\left[X X^{0}, X^{0}\right]$, and (c) $\left[X X^{0}, X^{-}\right]$, and autocorrelation functions from (d) $\left[X^{0}, X^{0}\right]$ and (e) $\left[X^{-}, X^{-}\right]$ configurations are summarized. All these functions were measured under an identical excitation condition. The crosscorrelation function basically exhibits asymmetric line shape because the underlying physical process for [start, stop] $=[\alpha, \beta]$ and the reversed configuration is not equivalent. ${ }^{24,26,28-30}$ For example, in Fig. 2(a), the asymmetric $g^{(2)}(\tau)$ observed in the positive (negative) $\tau$ region for $\left[X^{0}, X^{-}\right]$is due to the difference in the carrier-population rate to the $\left|X^{-}\right\rangle\left(\left|X^{0}\right\rangle\right)$ state after $X^{0}\left(X^{-}\right)$photon emission. ${ }^{26-28,30}$

For the $\left[X X^{0}, X^{0}\right]$ configuration, a high bunching peak with decay in the positive $\tau$ region is observed in Fig. 2(b). This means that the $X^{0}$ photon is much frequently found after the $X X^{0}$ emission compared with a Poisson distribution due to the $\left|X X^{0}\right\rangle \rightarrow\left|X^{0}\right\rangle \rightarrow|\mathrm{Vac}\rangle$ cascade transition after each excitation event, ${ }^{25,26,28-30}$ and the recombination rate of $X^{0}$ determines the decay rate of the bunching peak for $\tau>0$. As for the $\left[X X^{0}, X^{-}\right]$configuration, a weak bunching is observed in the $\tau>0$ region as shown in Fig. 2(c). In this case, bunching appears only when the QD is subject to electron capture at the dwell time of the $\left|X^{0}\right\rangle$ after $X X^{0}$ emission. Hence this bunching suggests that single-electron capture predominantly occurs in the present QD. ${ }^{28,29}$

In Figs. 2(d) and 2(e), autocorrelation functions with symmetric line shapes were obtained. For the $\left[X^{0}, X^{0}\right]$ configuration, a broad bunched structure is superposed on a conventional antibunching structure. This is a signature that the $\left|X^{0}\right\rangle$ and $|\mathrm{Vac}\rangle$, which are responsible for the $X^{0}$ photon emission compose an open system for carriers, as reported in Refs. 17-27. In contrast, no bunching was observed for the $\left[X^{-}, X^{-}\right]$. Assume that two states $\left|s_{1}\right\rangle$ and $\left|s_{2}\right\rangle$ are exclusively populated and these are coupled by carrier transfer. When $\left|s_{1}\right\rangle \rightarrow\left|s_{2}\right\rangle$ preferentially occurs against $\left|s_{2}\right\rangle \rightarrow\left|s_{1}\right\rangle$, superposed bunching appears in the autocorrelation function for $\left|s_{1}\right\rangle$ because population in the QD shifts to $\left|s_{2}\right\rangle$ as delay time passes. 




FIG. 3. (Color online) Five-level scheme used for the rateequation analysis of charge-transfer dynamics. $G, \gamma_{L}, \gamma_{\text {in(out) }}$, and $\gamma_{\text {in(out) }}^{\prime}$ are exciton photogeneration rate, radiative recombination rate of the line $L\left(L=X^{0}, X X^{0}, X^{-}\right)$, single-electron capture (escape) rate, and single-hole capture (escape) rate, respectively. For the excitation quasiresonantly to $X^{0}, G$ 's in $\left|e^{-}\right\rangle \rightarrow\left|X^{-}\right\rangle$and $\left|X^{0}\right\rangle \rightarrow\left|X X^{0}\right\rangle$ processes are set to zero. Population in each state is denoted by $n_{i}$ $(i=1-5)$.

Therefore, by comparing the autocorrelation functions for $X^{0}$ [see Fig. 2(d)] and $X^{-}$[see Fig. 2(e)], the preferential state variation from $\left|X^{0}\right\rangle$ to $\left|X^{-}\right\rangle$via single-electron capture to the QD is endorsed. In addition, the single-electron-capture rate is sensitively reflected on the decay constant for the bunching peak appeared in the $\left[X^{0}, X^{0}\right]$ toward steady state as $|\tau| \rightarrow \infty$.

To numerically analyze carrier-transfer dynamics in the QD, we performed rate-equation analysis using a five-level scheme. Figure 3 shows a schematic of the states involved and the possible transitions between them occurring via excitation, recombination, and single-carrier capture/escape. From this model, we can formulate a set of rate equations as follows:

$$
\begin{aligned}
\frac{d n_{1}(t)}{d t}= & -\left(G+\gamma_{\text {in }}\right) n_{1}(t)+\gamma_{X^{0}} n_{2}(t)+\gamma_{\text {out }} n_{4}(t), \\
\frac{d n_{2}(t)}{d t}= & G n_{1}(t)-\left(G+\gamma_{X^{0}}+\gamma_{\text {in }}+\gamma_{\text {out }}^{\prime}\right) n_{2}(t) \\
& +\gamma_{X X^{0}} n_{3}(t)+\gamma_{\text {in }}^{\prime} n_{4}(t)+\gamma_{\text {out }} n_{5}(t), \\
\frac{d n_{3}(t)}{d t}= & G n_{2}(t)-\left(\gamma_{X X^{0}}+\gamma_{\text {out }}^{\prime}\right) n_{3}(t)+\gamma_{\text {in }}^{\prime} n_{5}(t), \\
\frac{d n_{4}(t)}{d t}= & \gamma_{\text {in }} n_{1}(t)+\gamma_{\text {out }}^{\prime} n_{2}(t)-\left(G+\gamma_{\text {in }}^{\prime}+\gamma_{\text {out }}\right) n_{4}(t) \\
& +\gamma_{X^{-}} n_{5}(t), \\
\frac{d n_{5}(t)}{d t}= & \gamma_{\text {in }} n_{2}(t)+\gamma_{\text {out }}^{\prime} n_{3}(t)+G n_{4}(t) \\
& -\left(\gamma_{X^{-}}+\gamma_{\text {in }}^{\prime}+\gamma_{\text {out }}\right) n_{5}(t),
\end{aligned}
$$

where $n_{1}(t), n_{2}(t), n_{3}(t), n_{4}(t)$, and $n_{5}(t)$ are population probabilities for the vacuum state $|\mathrm{Vac}\rangle,\left|X^{0}\right\rangle,\left|X X^{0}\right\rangle$, singleelectron state $\left|e^{-}\right\rangle$, and the $\left|X^{-}\right\rangle$state at time $t$, respectively. The experimentally obtained lifetimes for $X^{0}, X X^{0}$, and $X^{-}$ emission lines in Fig. 1(c) were used for $1 / \gamma_{X^{0}}, 1 / \gamma_{X X^{0}}$, and $1 / \gamma_{X^{-}}$, respectively.

Based on the time-dependent solutions of Eq. (1), we have simulated the second-order photon correlation functions as a function of delay time. $g^{(2)}(\tau)$ in [start, stop] $=[\alpha, \beta]$ configurations are given by $n_{\beta}(t)\left[n_{\alpha}(t)\right]$ under the condition $n_{\alpha^{\prime}}(0)\left[n_{\beta^{\prime}}(0)\right]=1$ for $\tau \geqslant 0 \quad(\tau<0)$, where $n_{\alpha^{\prime}}$ $\left(n_{\beta^{\prime}}\right)$ is the population of state $\left|\alpha^{\prime}\right\rangle\left(\left|\beta^{\prime}\right\rangle\right)$ directly coupled with $|\alpha\rangle(|\beta\rangle)$ by optical transition of $|\alpha\rangle \rightarrow\left|\alpha^{\prime}\right\rangle$ $\left(|\beta\rangle \rightarrow\left|\beta^{\prime}\right\rangle\right){ }^{26,28,29}$ The red curves in Fig. 2 show the simulated results based on Eq. (1). The calculated curves are in good agreement with the experimental results. A used parameter set is $\vec{\zeta} \equiv\left(G, \gamma_{\text {in }}, \gamma_{\text {out }}, \gamma_{\text {in }}^{\prime}, \gamma_{\text {out }}^{\prime}\right)=$ $(0.055,0.280,0.030,0.030,0.020)$ in gigahertz, and $\vec{\zeta}$ is common for all correlation functions with the five configurations. For the present simulation, the response function of our setup and a baseline $(\leqslant 5 \%)$ caused by residual uncorrelated photons were taken into account.

Since $\gamma_{\text {in }}$ overwhelms other parameters in the present nonresonant excitation, the excitonic species in the QD are populated by single-electron capture rather than electron-hole capture. This finding is also supported by Fig. 1(a), in which $X^{-}$emission is distinctly observed. In contrast, $\gamma_{\text {in }}^{\prime}$ makes a minor contribution. Therefore the population of a positively charged exciton $\left(X^{+}\right)$state is considered to be inefficient, which is consistent with the lack of $X^{+}$emission line in the PL spectrum. We have found that the height of the bunching peak in $\left[X^{0}, X^{0}\right]$ is determined by the ratio $\gamma_{\text {in }} / \gamma_{\text {out }}$, and its decay rate is given by $\gamma_{\text {in }} \cdot \gamma_{\text {in }}$ simultaneously governs the rise rate for $\tau>0$ in $\left[X^{0}, X^{-}\right]$, while $\gamma_{\text {in }}^{\prime}$ governs the rise rate for $\tau<0$. These behaviors nearly follow the analytical solution obtained in Ref. 17, where the exciton transfer between two energy windows in a single emission line (i.e., charge state in the QD is fixed) is argued. In this case, processes connecting the upper and lower states, indicated by $\gamma_{\text {in }}^{\prime}$ and $\gamma_{\text {out }}^{\prime}$ (magenta arrows) in Fig. 3, are physically invalid, which helps in simplifying the model and enables analytical solutions to be found.

\section{Excitation-power dependence}

Figure 4(a) shows the examples of measured $g^{(2)}(\tau)$ in the $\left[X^{0}, X^{0}\right]$ configuration depending on excitation power. Here, the trace of $65 \mu \mathrm{W}$ is replotted from Fig. 2(d) for comparison.
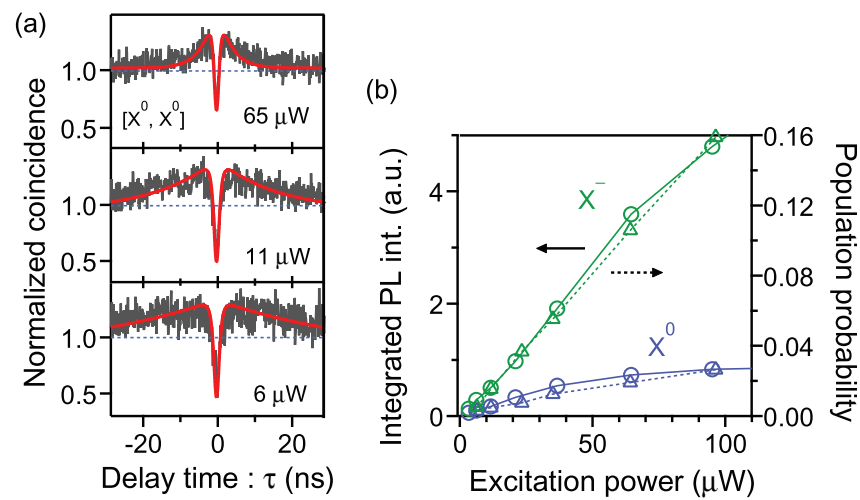

FIG. 4. (Color online) (a) Autocorrelation functions for the $X^{0}$ at three excitation powers. Decay of the bunching peak at $\tau \sim 0$ becomes slower as the excitation power decreases. (b) Steady-state solutions of the rate equations (triangles) compared with measured PL intensities for $X^{0}$ and $X^{-}$emissions (circles). Circles (triangles) indicate the integrated PL intensities (calculation). Right axis is obtained after appropriate scaling to the PL intensity. 
For all excitation powers, $g^{(2)}(\tau)$ exhibits clear bunching peaks at $\tau \sim 0$. This indicates that preferential single-electron capture to the QD remains unchanged with variations in the excitation power. However, the decay of the bunching becomes faster as increasing the excitation power. This suggests that the electrons to be captured to the QD originate from electron-hole pairs optically excited in GaAs barrier.

The rate-equation analysis was repeated for $g^{(2)}(\tau)$ measured at each excitation power in the same manner as that for the $65 \mu \mathrm{W}$ excitation discussed in the previous section. Owing to insufficient $X X^{0}$ intensity at excitation power below $65 \mu \mathrm{W}, G, \gamma_{\text {in(out) }}$, and $\gamma_{\text {in }}^{\prime}$ were determined to provide the best fit to the correlation functions obtained for configurations $\left[X^{0}, X^{0}\right],\left[X^{-}, X^{-}\right]$, and $\left[X^{0}, X^{-}\right]$(not shown). The steadystate solutions for $n_{2}(t)$ and $n_{5}(t)$ in Eq. (1) at each excitation power are given by the triangles in Fig. 4(b) and are compared with the PL intensities (circles) for the $X^{0}$ and $X^{-}$lines with an appropriate scaling.

For given a parameter set $\vec{\zeta}$ that explains measured $g^{(2)}(\tau)$ under a specific excitation power $P$, our rate-equation analysis show that $g^{(2)}(\tau)$ can well reproduced with $\rho \vec{\zeta}$ under other excitation power $P^{\prime}$, where the $\rho$ is a parameter to be determined to give the best fit to the $g^{(2)}(\tau)$ and is approximately given as $P^{\prime} / P$. This result suggests that the relative values between the parameters are nearly unchanged regardless of the excitation power, which is partly supported by the constant bunching peak heights in Fig. 4(a) determined by the $\gamma_{\text {in }} / \gamma_{\text {out }} \sim 9$ and also by the fact that $\gamma_{\text {in }}$ increases linearly with respect to the excitation power, as shown by red circles in Fig. 6. The preferential single-electron capture to the QD is probably influenced by the unbalanced relaxation rate of the photogenerated electrons and holes in the GaAs barrier region falling to the ground state in the QD. ${ }^{40-42}$

As discussed above, the underlying carrier dynamics around the QD was successfully analyzed, and an electron-transfer process was shown to be dominant. However, from a viewpoint of quantum emitter, this process hinders the stability of excitonic states populated in the QD. In this regard, suppressing the single-electron capture, which dominates the dynamics, can be beneficial. In the next section, we discuss the effect of excitation energy on this issue.

\section{Excitation-energy dependence}

We have investigated how excitation energy $\left(E_{\text {exc }}\right)$ affects carrier-transfer dynamics. The inset in Fig. 5(a) shows the $\mu$-PL excitation (PLE) spectrum for the $X^{0}$ emission located at $E_{X^{0}}=1.3014 \mathrm{eV}$. A prominent peak is clearly observed at $E_{\text {exc }}-E_{X^{0}}=34.6 \mathrm{meV}$, as indicated by the red arrow. This corresponds to the longitudinal optical (LO) phonon energy of the GaAs bulk. Under this quasiresonant excitation condition, the exclusive generation of $X^{0}$ is possible, especially for a weak excitation power, as shown in Fig. 5(a).

Figure 5(b) illustrates the autocorrelation functions for the $X^{0}$ emission line measured under three excitation powers. The bunching peaks at $\tau \sim 0$ are also shown, which suggests that single-electron capture is still dominant even under this quasiresonant excitation. However, the decay rate is much slower than that of nonresonant excitation [see Fig. 4(a)]. This is a clear manifestation of the strongly suppressed $\gamma_{\text {in }}$ under the
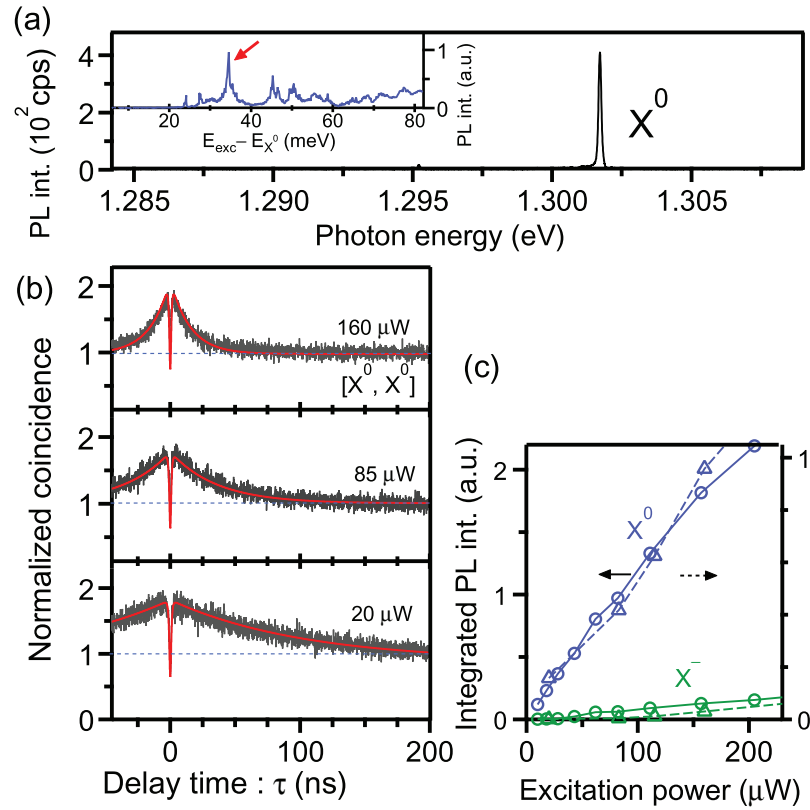

(c)

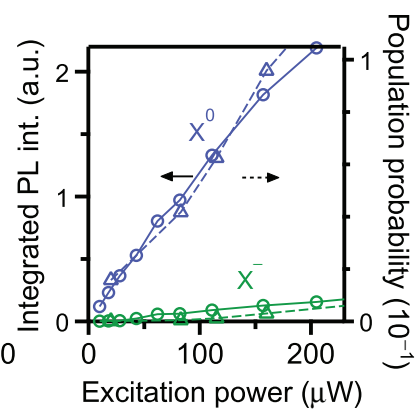

FIG. 5. (Color online) (a) $\mu$-PL spectrum under quasiresonant excitation with the same QD at $20 \mu \mathrm{W}$ excitation. $X^{0}$ is exclusively populated. $\mu$-PLE spectrum with $X^{0}$ emission is shown in the inset. (b) Autocorrelation functions for $X^{0}$ under quasiresonant excitation. Slower decay of the bunching peaks is distinct in comparison to nonresonant excitation. (c) Steady-state solutions (triangles) compared with measured PL intensities for $X^{0}$ and $X^{-}$emissions (circles). In contrast to Fig. 4(b), the $\left|X^{0}\right\rangle$ state is predominantly populated.

present quasiresonant excitation, and this result is consistent with the absence of $X^{-}$emission in the PL spectrum, as shown in Fig. 5(a).

For analyzing carrier dynamics under quasiresonant excitation, the preferential excitation of $X^{0}$ is assumed, and the excitation rates ( $G$ in Fig. 3) for the $\left|e^{-}\right\rangle \rightarrow\left|X^{-}\right\rangle$ and $\left|X^{0}\right\rangle \rightarrow\left|X X^{0}\right\rangle$ processes are set to zero. First, we consider $g^{(2)}(\tau)$ with five configurations at a relatively high excitation power of $160 \mu \mathrm{W}$ in the same manner as discussed in Fig. 2. The simulated results are in good agreement with the five configurations. An used parameter set is $\vec{\zeta}=$ $(0.150,0.040,0.008,0.015,0.008)$ in gigahertz, which gives the red curve in Fig. 5(b). It is clarified that $\gamma_{\text {in }}$ is much smaller than $G\left(\gamma_{\text {in }} \ll G\right)$, indicating that direct creation of $X^{0}$ in the QD after photoexcitation is the dominant process for the exciton population. This is in strong contrast to the case of nonresonant excitation, which leads to $\gamma_{\text {in }} \gg G$.

At lower excitation powers, the cross-correlation functions can no longer be measured. Hence, to discuss the dynamics under quasiresonant excitation, we focused on the bunching peak in the autocorrelation functions for $X^{0}$ and find the $\rho$ values at each excitation power. Consequently, the parameter $\vec{\zeta}$ describing the dynamics is uniquely obtained. The PL intensities of the $X^{0}$ and $X^{-}$lines are also consistently explained, as shown in Fig. 5(c). This is obtained by solving Eq. (1) for the steady-state population with the common parameter at each excitation power $\vec{\zeta}$ used in evaluating the $X^{0}$ autocorrelation functions. Note that the slight underestimation of $X^{-}$intensities is observed on closer examination. This occurs because in our model, the exclusive excitation via 


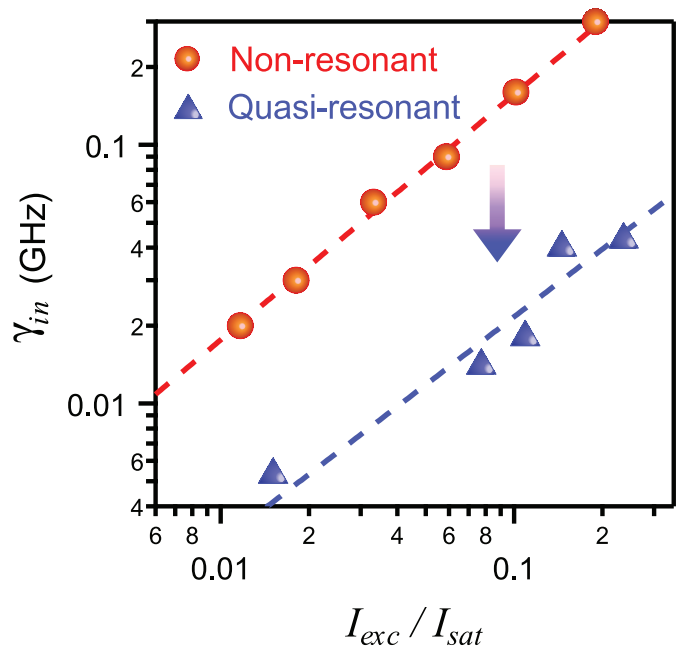

FIG. 6. (Color online) Summary of single-electron capture rates $\left(\gamma_{\text {in }}\right)$ evaluated under various excitation conditions. Transverse axis indicates an effective exciton population. Under quasiresonant excitation, $\gamma_{\text {in }}$ is suppressed by one order of magnitude. Dashed lines are guides to the eye.

one LO phonon, which couples only $|\mathrm{Vac}\rangle \rightarrow\left|X^{0}\right\rangle$, was assumed. Actually, including a few contributions $(\sim 3 \%)$ of $G$ in $\left|e^{-}\right\rangle \rightarrow\left|X^{-}\right\rangle$provides values that are considerably close to the measured PL intensities. This suggests that the electron-hole capture process in the dwell time of $\left|e^{-}\right\rangle$makes a finite contribution to the $X^{-}$emission, probably through the residual continuum state in the present $\mathrm{QD},{ }^{43}$ as observed in the PLE spectrum given in the inset of Fig. 5(a).

Figure 6 compares the $\gamma_{\text {in }}$ values analyzed under nonresonant (red circles) and quasiresonant (blue triangles) excitations. The horizontal axis indicates the sum of the $X^{0}$ and $X^{-}$intensities normalized by their total saturation intensity. This can be an appropriate measure for comparing carrier-capture rates under non and quasiresonant excitations, since the underlying processes could be sensitively affected by the effective exciton population in the QD. For the both excitation energies, $\gamma_{\text {in }}$ is approximately proportional to the laser power. However, by introducing quasiresonant excitation, the capture rate can be effectively suppressed by one order of magnitude compared with nonresonant excitation.

\section{CONCLUSIONS}

We have comprehensively investigated the carrier-transfer dynamics occurring in an excitonic system formed in a single InAs QD. The experimentally obtained second-order photon correlation functions were analyzed on the basis of a series of rate equations, which evaluate population flows between five exciton-related states, including single-carriercapture/escape processes. The validity of this approach was confirmed by its ability to evaluate correlation functions and steady-state populations with PL intensities measured under various excitation conditions.

The analysis enabled us to quantitatively evaluate the characteristic switching time of the excitonic charge states in the QD and provided a detailed understanding of the carriertransfer dynamics, which is essential for obtaining highly stable solid-state single-photon emitters without undesirable charge fluctuations. One of the most important findings was that the single-carrier-transfer rate is strongly suppressed by one order of magnitude under quasiresonant excitation.

\section{ACKNOWLEDGMENTS}

We would like to thank H. Sasakura and C. Hermannstädter for their fruitful discussions. This work was supported partly by Grants-in-Aid for Scientific Research (B) No. 24310084 and (S) No. 24226007 from the Ministry of Education, Cluture, Sports, Science and Technology (MEXT) and Strategic Information and Communications R\&D Promotion Programme (SCOPE) from the Ministry of Internal Affairs and Communications. *nakajima@es.hokudai.ac.jp

${ }^{1}$ D. Gammon, E. S. Snow, B. V. Shanabrook, D. S. Katzer, and

D. Park, Science 273, 87 (1996).

${ }^{2}$ N. Gisin, G. Ribordy, W. Tittel, and H. Zbinden, Rev. Mod. Phys.

74, 145 (2002).

${ }^{3}$ E. Knill, R. Laflamme, and G. J. Milburn, Nature (London) 409, 46 (2001).

${ }^{4}$ P. Michler, A. Kiraz, C. Becher, W. V. Schoenfeld, P. M. Petroff, L. Zhang, E. Hu, and A. Imamoğlu, Science 290, 2282 (2000).

${ }^{5}$ V. Zwiller, H. Blom, P. Jonsson, N. Panev, S. Jeppesen, T. Tsegaye, E. Goobar, M.-E. Pistol, L. Samuelson, and G. Bjork, Appl. Phys. Lett. 78, 2476 (2001).

${ }^{6}$ P. Ester, L. Lackmann, S. Michaelisde de Vasconcellos, M. C. Hübner, A. Zrenner, and M. Bicheler, Appl. Phys. Lett. 91, 111110 (2007).

${ }^{7}$ J. Claudon, J. Bleuse, N. S. Malik, M. Bazin, P. Jaffrennou, N. Gregersen, C. Sauvan, P. Lalanne, and J.-M. Gérard, Nat. Photon. 4, 174 (2010).

${ }^{8}$ H. Nakajima, H. Kumano, H. Iijima, and I. Suemune, Appl. Phys. Lett. 101, 161107 (2012).
${ }^{9}$ D. Fattal, K. Inoue, J. Vučković, C. Santori, G. S. Solomon, and Y. Yamamoto, Phys. Rev. Lett. 92, 037903 (2004).

${ }^{10}$ R. M. Stevenson, R. J. Young, P. Atkinson, K. Cooper, D. A. Ritchie, and A. J. Shields, Nature (London) 439, 178 (2006).

${ }^{11}$ N. Akopian, N. H. Lindner, E. Poem, Y. Berlatzky, J. Avron, D. Gershoni, B. D. Gerardot, and P. M. Petroff, Phys. Rev. Lett. 96, 130501 (2006).

${ }^{12}$ R. Hafenbrak, S. M. Ulrich, P. Michler, L. Wang, A. Rastelli, and O. G. Schmidt, New J. Phys. 9, 315 (2007).

${ }^{13}$ A. Dousse, J. Suffczynski, A. Beveratos, O. Krebs, A. Lematre, I. Sagnes, J. Bloch, P. Voisin, and P. Senellart, Nature (London) 466, 217 (2010).

${ }^{14}$ M. Ghali, K. Ohtani, Y. Ohno, and H. Ohno, Nat. Commun. 3, 661 (2012).

${ }^{15}$ R. Trotta, E. Zallo, C. Ortix, P. Atkinson, J. D. Plumhof, J. van den Brink, A. Rastelli, and O. G. Schmidt, Phys. Rev. Lett. 109, 147401 (2012).

${ }^{16}$ T. Kuroda, T. Mano, N. Ha, H. Nakajima, H. Kumano, B. Urbaszek, M. Jo, M. Abbarachi, Y. Sakuma, K. Sakoda, I. Suemune, X. Marie, and T. Amand, Phys. Rev. B 88, 041306(R) (2013). 
${ }^{17}$ G. Sallen, A. Tribu, T. Aichele, R. André, L. Besombes, C. Bougerol, M. Richard, S. Tatarenko, K. Kheng, and J.-Ph. Poizat, Nat. Photon. 4, 696 (2010).

${ }^{18}$ G. Sallen, A. Tribu, T. Aichele, R. André, L. Besombes, C. Bougerol, M. Richard, S. Tatarenko, K. Kheng, and J.-Ph. Poizat, Phys. Rev. B 84, 041405(R) (2011).

${ }^{19}$ M. Abbarchi, T. Kuroda, T. Mano, M. Gurioli, and K. Sakoda, Phys. Rev. B 86, 115330 (2012).

${ }^{20}$ J. Wolters, N. Sadzak, A. W. Schell, T. Schröder, and O. Benson, Phys. Rev. Lett. 110, 027401 (2013).

${ }^{21}$ C. Santori, D. Fattal, J. Vučković, G. S. Solomon, and Y. Yamamoto, Nature (London) 419, 594 (2002).

${ }^{22}$ K. Takemoto, Y. Nambu, T. Miyazawa, K. Wakui, S. Hirose, T. Usuki, M. Takatsu, N. Yokoyama, K. Yoshino, A. Tomita, S. Yorozu, Y. Sakuma, and Y. Arakawa, Appl. Phys. Express 3, 092802 (2010).

${ }^{23}$ T. M. Babinec, B. J. M. Hausmann, M. Khan, Y. Zhang, J. R. Maze, P. R. Hemmer, and M. Lončar, Nat. Nanotechnol. 5, 195 (2010).

${ }^{24}$ S. M. Ulrich, M. Benyoucef, P. Michler, N. Baer, P. Gartner, F. Jahnke, M. Schwab, H. Kurtze, M. Bayer, S. Fafard, Z. Wasilewski, and A. Forchel, Phys. Rev. B 71, 235328 (2005).

${ }^{25}$ C. Santori, D. Fattal, J. Vučković, G. S. Solomon, E. Waks, and Y. Yamamoto, Phys. Rev. B 69, 205324 (2004).

${ }^{26}$ G. Sallen, A. Tribu, T. Aichele, R. André, L. Besombes, C. Bougerol, S. Tatarenko, K. Kheng, and J.-Ph. Poizat, Phys. Rev. B 80, 085310 (2009).

${ }^{27}$ B. Piętka, J. Suffczyński, M. Goryca, T. Kazimierczuk, A. Golnik, P. Kossacki, A. Wysmolek, J. A. Gaj, R. Stępniewski, and M. Potemski, Phys. Rev. B 87, 035310 (2013).

${ }^{28}$ M. H. Baier, A. Malko, E. Pelucchi, D. Y. Oberli, and E. Kapon, Phys. Rev. B 73, 205321 (2006).

${ }^{29}$ J. Suffczyński, T. Kazimierczuk, M. Goryca, B. Piechal, A. Trajnerowicz, K. Kowalik, P. Kossacki, A. Golnik, K. P. Korona, M. Nawrocki, J. A. Gaj, and G. Karczewski, Phys. Rev. B 74, 085319 (2006).
${ }^{30}$ A. Kiraz, S. Fälth, C. Becher, B. Gayral, W. V. Schoenfeld, P. M. Petroff, L. Zhang, E. Hu, and A. Imamoğlu, Phys. Rev. B 65, 161303(R) (2002).

${ }^{31}$ X. Liu, T. Asano, S. Odashima, H. Nakajima, H. Kumano, and I. Suemune, Appl. Phys. Lett. 102, 131114 (2013).

${ }^{32}$ H. Kumano, H. Nakajima, H. Iijima, S. Odashima, Y. Matsuo, K. Ijiro, and I. Suemune, Appl. Phys. Express 6, 062801 (2013).

${ }^{33}$ H. Kumano, H. Nakajima, S. Ekuni, Y. Idutsu, H. Sasakura, and I. Suemune, Adv. Math. Phys. (2010) 391607.

${ }^{34}$ R. Hnabury-Brown and R. Q. Twiss, Nature (London) 178, 1447 (1956).

${ }^{35}$ J. M. Smith, P. A. Dalgarno, R. J. Warburton, A. O. Govorov, K. Karrai, B. D. Gerardot, and P. M. Petroff, Phys. Rev. Lett. 94, 197402 (2005).

${ }^{36}$ S. Strauf, N. G. Stoltz, M. T. Rakher, L. A. Coldren, P. M. Petroff, and D. Bouwmeester, Nat. Photon. 1, 704 (2007).

${ }^{37}$ M. Abbarchi, T. Kuroda, T. Mano, K. Sakoda, C. A. Mastrandrea, A. Vinattieri, M. Gurioli, and T. Tsuchiya, Phys. Rev. B 82, 201301(R) (2010).

${ }^{38}$ R. Seguin, A. Schliwa, T. D. Germann, S. Rodt, K. Pötschke, A. Strittmater, U. W. Pohl, D. Bimberg, M. Winkelnkemper, T. Hammerschmidt, and P. Krazer, Appl. Phys. Lett. 89, 263109 (2006).

${ }^{39}$ M. Bayer, A. Kuther, A. Forchel, A. Gorbunov, V. B. Timofeev, F. Schäfer, J. P. Reithmaier, T. L. Reinecke, and S. N. Walck, Phys. Rev. Lett. 82, 1748 (1999).

${ }^{40}$ U. Woggon, H. Giessen, F. Gindele, O. Wind, B. Fluegel, and N. Peyghambarian, Phys. Rev. B 54, 17681 (1996).

${ }^{41}$ T. S. Sosnowski, T. B. Norris, H. Jiang, J. Singh, K. Kamath, and P. Bhattacharya, Phys. Rev. B 57, R9423 (1998).

${ }^{42}$ D. A. Yarotski, R. D. Averitt, N. Negre, S. A. Crooker, A. J. Taylor, G. P. Donati, A. Stintz, L. F. Lester, and K. J. Malloy, J. Opt. Soc. Am. B 19, 1480 (2002).

${ }^{43}$ Y. Toda, O. Moriwaki, M. Nishioka, and Y. Arakawa, Phys. Rev. Lett. 82, 4114 (1999). 